\title{
RECONOCIMIENTO DE PALABRAS EN ESPAÑOL EN UNA TAREA DE DECISIÓN LÉXICA VISUAL CON PSEUDOHOMÓFONOS
}

\section{RECOGNITION OF WORDS IN SPANISH IN A VISUAL LEXICAL DECISION TASK WITH PSEUDOHOMOPHONES}

\author{
Micaela Difalcis \\ Consejo Nacional de Investigaciones Científicas y Técnicas, Argentina \\ micaeladifalcis@gmail.com \\ Samanta Leiva \\ Universidad de Buenos Aires, Argentina \\ samantaleiva1@gmail.com \\ Aldo Ferreres \\ Universidad de Buenos Aires, Argentina \\ aldoferreres@gmail.com \\ Valeria Abusamra \\ Consejo Nacional de Investigaciones Científicas y Técnicas, Argentina \\ vabusamra@gmail.com
}

\begin{abstract}
Resumen:
Los modelos de reconocimiento de palabras escritas aisladas presentan divergencias acerca de si la activación fonológica es automática en la lectura silente o si se trata de un procesamiento posterior al acceso léxico-semántico. El efecto de pseudohomofonía se ha analizado de manera recurrente en diferentes lenguas para estudiar el grado de mediación fonológica. Sin embargo, son pocos los estudios llevados a cabo en lengua española. El objetivo del presente trabajo ha sido investigar el efecto de pseudohomofonía en una tarea de decisión léxica visual (tanto en el análisis de los tiempos de reacción como de la tasa de error) en la que, por primera vez en lengua española variedad rioplatense, las variables léxicas y subléxicas de los estímulos han sido exhaustivamente controladas. Hallamos un efecto de pseudohomofonía en el análisis de la tasa de error pero no en el análisis de los tiempos de reacción. Esto último es evidencia a favor de una participación no automática de los procesos fonológicos en el reconocimiento de palabras aisladas.
\end{abstract}

Palabras clave: decisión léxica, pseudohomófonos, español, lectura

\begin{abstract}
:
The models of word's recognition present divergences about whether the phonological activation is automatic in the silent reading or if it is a post-lexical-semantic access processing. The effect of pseudohomophony has been analyzed repeatedly in different languages to study the degree of phonological mediation. However, there are few studies carried out in Spanish. The aim of the present work was to investigate the effect of pseudohomophony in a visual lexical decision task (both in the analysis of the reaction times and the error rate) in which, for the first time in Spanish (variety of Río de la Plata), the lexical and sub-lexical variables of the stimuli have been exhaustively controlled. We found an effect of pseudohomophony in the analysis of the error rate but not in the analysis of reaction times. The latter would contribute to a nonautomatic participation of phonological processes in the recognition of words.
\end{abstract}

Keywords: lexical decision, pseudohomophones, Spanish, reading 
Artículo. Micaela Difalcis, Samanta Leiva, Aldo Ferreres, Valeria Abusamra. Reconocimiento de palabras en español en una tarea de decisión léxica visual con pseudohomófonos

\section{Introducción}

Los estudios sobre los procesos cognitivos implicados en el reconocimiento visual de una palabra aislada asumen, en su mayoría, la existencia de dos mecanismos de procesamiento: una vía léxica y una vía subléxica o "fonológica" (Coltheart et al., 2001; Coslett, 2003; Ellis y Young, 1988). Ambas vías se ponen en funcionamiento cuando a un sujeto se le presenta un estímulo ortográfico pero el grado de contribución de cada una a la lectura variará según el tipo de estímulo -si es una palabra existente o no-, la lengua -el procesamiento no será igual en lenguas con altos niveles de correspondencias entre grafemas y fonemas, llamadas "transparentes", que en lenguas con bajos niveles, llamadas "opacas" (Kwok et al., 2017) - y el grado de destreza del lector -no será igual el nivel de participación de una u otra vía en lectores principiantes que en lectores avanzados (Kuperman y Van Dyke, 2011; Andrews, 2012; Adelman, et al., 2014)- .

La tarea de decisión léxica visual (DL) se ha utilizado de manera recurrente en las investigaciones psicolingüísticas para estudiar el reconocimiento visual de las palabras escritas aisladas (Perea y Rosa, 1999). En ella, los participantes deben decidir de la manera más rápida y precisa posible si el estímulo que se les presenta es una palabra existente en su lengua o si, por el contrario, no lo es. Las conclusiones de estos estudios se obtienen, básicamente, a partir de dos tipos de información: los tiempos de reacción (TRs), es decir, los milisegundos transcurridos desde la presentación del estímulo hasta la decisión ejecutada por el participante; y, por otro lado, la tasa de error producida. Para estudiar el funcionamiento y la interacción de ambas vías de reconocimiento a través del paradigma de la decisión léxica visual, se manipulan diferentes variables en los experimentos. Los efectos más estudiados son el de lexicalidad (las palabras se reconocen más rápidamente que las no-palabras) y el de frecuencia (las palabras de alta frecuencia se reconocen más rápidamente que las de baja frecuencia).

Otra variable que ha generado interés es la pseudohomofonía. Los pseudohomófonos (PSHs) son cadenas de letras que forman una palabra no existente pero cuya forma fonológica es idéntica a la de una palabra existente (por ejemplo, el pseudohomófono "voseto" cuya forma fonológica es igual a la palabra "boceto"). El efecto de pseudohomofonía se puede observar en mayores latencias para rechazar PSHs 
en una tarea de decisión léxica visual que para rechazar una no-palabra que no posee una forma fonológica correspondiente a una palabra de la lengua (por ejemplo, “bofeto"). Fue reportado por primera vez por Rubenstein, Lewis y Rubenstein (1971) quienes hallaron dicho efecto en un estudio realizado en angloparlantes. Además, encontraron interacción con la frecuencia de la palabra a partir de la cual se derivaba el PSH (las latencias eran mayores para los estímulos cuyas palabra base eran de baja frecuencia) ${ }^{1}$.

El análisis del efecto de pseudohomofonía permite estudiar cuál es el papel que cumple la fonología en el reconocimiento visual de las palabras y, por lo tanto, conocer el grado de implicancia de cada una de las vías de lectura en los modelos de doble ruta. Si bien los investigadores acuerdan en que la información fonológica posee un rol importante tanto en el procesamiento del lenguaje oral como el lenguaje escrito, es aún objeto de debate el grado de activación en lectores expertos (Hawelka et al., 2013; Ziegler et al., 2014; Frisson et al., 2014) y, también, si el procesamiento fonológico de recodificación se activa de manera automática al guiar el acceso a las presentaciones léxico-semánticas. Existen dos puntos de vista principales sobre cómo obtenemos acceso al significado de las palabras en la lectura silenciosa. La hipótesis de acceso directo establece que existe una vía de la ortografía al significado (Seidenberg, 1985) y que, si la fonología influye de alguna manera en la lectura silenciosa, se trataría de un procesamiento posterior al acceso del significado. Por el contrario, la hipótesis de la mediación fonológica (Van Orden, 1987) sostiene que la activación fonológica es una condición necesaria para acceder al significado de las palabras. En consecuencia, se supone que la fonología se activa de manera automática antes del acceso al léxico (para revisiones ver Berent y Perfetti, 1995; Frost, 1998)

La mayor parte de los estudios sobre el efecto de pseudohomofonía se han llevado a cabo en participantes de lengua inglesa y también se ha reportado un efecto inverso entre latencias de lectura y decisión léxica: en tanto los PSHs se leen en voz alta más rápido que las no-palabras, estas son rechazadas más rápidamente que los PSHs (Coltheart et al., 1977; Besner y Davelaar, 1983; McCann y Besner, 1987; Taft y Russell, 1992; Seidenberg et al., 1996; Borowsky y Masson, 1999). Este fenómeno también se ha estudiado en otras lenguas como el francés (Grainger, Spinelli y Ferrand, 2000; Ferrand y Grainger, 2003) y el alemán (Ziegler, Jacobs y Klüppel, 2001; Braun et

\footnotetext{
${ }^{1}$ A partir de ahora, y para facilitar la lectura, siempre que mencionemos "PSH de alta frecuencia" o "PSH de baja frecuencia" estaremos haciendo referencia a la frecuencia de la palabra base de dicho PSH debido a que, por tratarse de palabras no existentes en la lengua, los PSHs no poseen frecuencia de aparición propia.
} 
al., 2015). Ziegler, Jacobs y Klüppel (2001) estudiaron el efecto de pseudohomofonía en lengua alemana y replicaron la desventaja de los PSHs respecto de las no-palabras en la decisión léxica y encontraron que la ventaja de los PSHs para la lectura en voz alta se relacionaba inversamente con la frecuencia de la palabra base: la ventaja era mayor cuando el PSH se derivaba de una palabra no frecuente que cuando se derivaba de una palabra frecuente. Estos autores interpretaron sus resultados proponiendo la intervención de dos mecanismos en la resolución de las tareas. En la tarea de decisión léxica, el PSH activaría un "mecanismo de verificación" consistente en la comparación de la información ortográfica del estímulo con la representación ortográfica activada por la forma fonológica activada por el PSH. La verificación dará como resultado mayores latencias para el rechazo de los PSH en la tarea de decisión léxica, es decir, una desventaja respecto del rechazo de las no-palabras que no poseen $\mathrm{y}$, por tanto, no activan ninguna representación ortográfica que ponga en marcha el mecanismo de verificación. Por su parte, en la tarea de lectura en voz alta, el PSH activaría una representación fonológica ya existente en el lexicón fonológico lo que representa una ventaja frente a las no-palabras que no la poseen.

Más recientemente, Barca et al. (2017) llevaron a cabo una tarea de decisión léxica con PSHs con el objetivo de estudiar la contribución específica de la información fonológica en el reconocimiento visual de palabras en lengua francesa. La novedad respecto de estudios anteriores realizados en esta lengua consistió en manipular la variable frecuencia de las palabras base de los PSHs. De esta manera, podrían determinar si el rechazo de los PSHs se lleva a cabo en el nivel léxico o a través del mecanismo de verificación. Si las latencias para los PSHs de alta frecuencia fueran mayores que para los PSHs de baja frecuencia, el mecanismo de decisión estaría desarrollándose a nivel lexical debido a que los niveles de activación de las palabras de alta frecuencia son mayores y, por lo tanto, más difíciles de inhibir. En cambio, en caso de obtener el patrón opuesto (mayores latencias para PSHs de baja frecuencia que para los de alta frecuencia), se pondría en funcionamiento el mecanismo de verificación en el que el alto grado de activación de una palabra de alta frecuencia conllevaría a una verificación más rápida y eficiente. Sin embargo, los autores no solo no hallaron diferencias significativas entre PSHs de alta y baja frecuencia, sino que los participantes mostraron un mejor rendimiento en PSHs que en no-palabras. Estos resultados fueron adjudicados al diseño adoptado para conformar la lista de estímulos: los autores no 
incluyeron homófonos heterógrafos (dos palabras con la misma forma fonológica pero distinta forma ortográfica) y esto, según ellos, podría haber condicionado la estrategia de lectura de los participantes (basada en un procesamiento fonológico más profundo por parte los sujetos).

En español, si bien recientemente se ha estudiado el efecto de frecuencia y lexicalidad en tareas de lectura en voz alta de palabras aisladas (Difalcis et al., 2018), solo Cuetos y Domínguez (2002) han estudiado el efecto de pseudohomofonía en tareas de lectura y decisión léxica visual, trabajo que, según nuestro conocimiento, no ha sido replicado aún. Estos autores encontraron un efecto cruzado: mientras los PSHs mostraban una desventaja (mayores latencias) respecto de las no-palabras en la decisión léxica, exhibían ventaja (menores latencias) en la lectura en voz alta. Los autores concluyeron que la pseudohomofonía es excitatoria en la tarea de lectura en voz alta e inhibitoria en la tarea de decisión léxica. El efecto excitatorio en la lectura se debería a que los PSHs activan una forma fonológica prexistente que las no-palabras no poseen. Por su parte, el efecto inhibitorio en decisión léxica se debería a la interferencia generada por la activación de la forma ortográfica correspondiente a la forma fonológica del PSH que debería ser inhibida para producir una respuesta correcta. Este trabajo, pionero en español, presenta la limitación de la falta de control de ciertas variables lexicales (como la cantidad de vecinos ortográficos) y sublexicales (frecuencias de bigramas) de los estímulos debida a la inexistencia, en ese momento, de diccionarios psicolingüísticos adecuados.

En el contexto del estudio limitado del efecto de pseudohomofonía en lengua española, el objetivo del presente trabajo fue estudiar dicho efecto en el español a través del diseño de una tarea de decisión léxica visual con PSHs en la que se han controlado de manera exhaustiva las variables léxicas y subléxicas de los estímulos. Además, hemos utilizado un diseño diferente para crear los PSHs y las no-palabras respecto de estudios anteriores llevados a cabo en español. Esperamos que, por otro lado, el diseño de la tarea pueda, eventualmente, ser aplicada a poblaciones clínicas para valorar la funcionalidad de las rutas léxica y no léxica de lectura (Ferreres et al., 2005; 2012).

Las hipótesis fueron que, en cuanto a los tiempos de reacción, los PSHs presentarán mayores latencias que las no-palabras y que, a su vez, las no-palabras presentarán mayores latencias que las palabras. En cuanto al análisis de los errores, se espera que los participantes cometan más errores con los PSHs que con las no-palabras y más con las no-palabras que con las palabras (Cuetos y Domínguez, 2002). Sin 
Artículo. Micaela Difalcis, Samanta Leiva, Aldo Ferreres, Valeria Abusamra. Reconocimiento de palabras en español en una tarea de decisión léxica visual con pseudohomófonos

embargo, en este último caso, no se esperan diferencias significativas Tanto en el análisis de los TRs como de los errores, estudiaremos si el efecto de frecuencia incide en el rendimiento de los sujetos y esperamos que los estímulos de alta frecuencia presenten TRs más cortos y una tasa de error más baja que los estímulos de baja frecuencia.

\section{Método}

\section{Participantes}

Noventa y un estudiantes (72 mujeres y 19 varones) del primer año de la carrera de Psicología de la Universidad de Buenos Aires participaron del experimento. Todos eran hablantes nativos del español y reportaron visión normal o compensada mediante anteojos. La media de edad fue de 22.1 (5.03), con un rango de 18 a 49 años. La media de escolaridad en años fue de 14.9 (1.51).

\section{Estímulos}

Se diseñó una prueba de decisión léxica con 55 palabras, 55 no-palabras y 55 PSHs. Para eso, se conformaron dos listas (A y B) de 55 palabras cada una, todos sustantivos sin tilde. La mitad de las palabras de cada una de las listas fue de alta frecuencia (entre 50,36 y 474,46) y la otra mitad, de baja frecuencia (entre 0,18 y 5,18). Ambas listas fueron emparejadas en variables léxicas (frecuencia y cantidad de vecinos ortográficos) y subléxicas (longitud en letras y frecuencia de bigramas token y type). La frecuencia fue considerada por cinco millones de palabras (base LEXESP) y todos los datos fueron extraídos de la base Busca Palabras (Davis y Perea, 2005).

Construcción de no-palabras y pseudohomófonos

Las 55 palabras de la lista A fueron incluidas como parte de la lista experimental y las 55 no-palabras y los 55 PSHs fueron conformados modificando una sola letra de cada una de las 55 palabras de la lista B. Por ejemplo, de la palabra de la lista B "noticia" se creó la no-palabra "notifia" y el PSH "noticia" (ver Apéndice). En todos los casos, se modificó la misma consonante de la palabra de la lista B para crear su correspondiente no-palabra y PSH. Los cambios para la creación de los PSHs se aplicaron modificando grafemas que en el español variedad rioplatense presentan homofonía heterográfica (/b/ > B-V, /s/ > C-S-Z, /x/ > G-J, /y/ > Y-LL) ubicados en la primera (21 estímulos), segunda (24 estímulos) o tercera sílaba (10 estímulos) de la palabra. Además, se utilizaron 55 palabras de relleno de frecuencia media para 
equilibrar la cantidad de palabras con la de no-palabras y PSH; los tiempos de reacción para las palabras de relleno no fueron considerados para el análisis de los resultados.

Por último, se conformó una lista de 36 estímulos distintos (12 palabras, 12 nopalabras y 12 PSHs) para ser utilizados como lista de ensayo. Las no-palabras y los PSHs de la lista de ensayo se construyeron siguiendo los mismos criterios que los de la prueba experimental.

\section{$\underline{\text { Procedimiento para el control de variables }}$}

En la Tabla 1 se informan los estadísticos descriptivos de las variables equilibradas y controladas. Para asegurar el correcto control de las variables de los estímulos, se realizó, en cada caso, las comparaciones pertinentes con la prueba $t$ para muestras independientes. En primer lugar, los resultados mostraron diferencias estadísticamente significativas entre las medias de las frecuencias de los estímulos de alta y baja frecuencia de las palabras de la lista A $(t=6.290,26.023 \mathrm{gl}, p<.001)$ y las palabras de la lista B $(t=11.363,27.176 \mathrm{gl}, \mathrm{p}<.001)$. Por otro lado, no se observaron diferencias entre las palabras de la lista A y B en longitud de letras $(t=-0.293,108 \mathrm{gl}, p$ $=.770)$, frecuencia de bigramas token $(t=1.467,108 \mathrm{gl}, \mathrm{p}=.145)$ y type $(t=-0.228$, $108 \mathrm{gl}, p=.820)$, cantidad de vecinos ortográficos $(t=1.265,108 \mathrm{gl}, p=.209)$ ni en la frecuencia media de las palabras de alta $(t=0.094,53 \mathrm{gl}, p=.925)$ y baja frecuencia $(t$ $=0.948,53 \mathrm{gl}, p=.347$ ). Además, no hubo diferencias significativas entre las palabras de la lista A y las no-palabras en la longitud de letras $(t=0.099,108 \mathrm{gl}, p=.921)$ y la frecuencia de bigramas token $(t=1.167,108 \mathrm{gl}, p=.246)$ y type $(t=-0.320,108 \mathrm{gl}, p=$ .750). Finalmente, no hubo diferencias entre las palabras de la lista A y los PSHs en la longitud de letras $(t=-0.196,108 \mathrm{gl}, p=.845)$ y la frecuencia de bigramas type $(t=$ $0.286,108 \mathrm{gl}, \mathrm{p}=.776)$.

\section{Tabla 1. Principales características de los estímulos}

\begin{tabular}{|c|c|c|c|c|c|c|c|c|c|c|c|c|c|c|c|c|}
\hline & \multicolumn{4}{|c|}{ Palabras (Lista A) } & \multicolumn{4}{|c|}{ Palabras (Lista B) } & \multicolumn{4}{|c|}{ No-palabras } & \multicolumn{4}{|c|}{ Pseudohomófonos } \\
\hline & \multicolumn{2}{|c|}{$\begin{array}{c}\text { Alta } \\
\text { frecuencia }\end{array}$} & \multicolumn{2}{|c|}{$\begin{array}{c}\text { Baja } \\
\text { frecuencia }\end{array}$} & \multicolumn{2}{|c|}{$\begin{array}{c}\text { Alta } \\
\text { frecuencia }\end{array}$} & \multicolumn{2}{|c|}{$\begin{array}{c}\text { Baja } \\
\text { frecuencia }\end{array}$} & \multicolumn{2}{|c|}{$\begin{array}{c}\text { Alta } \\
\text { frecuencia }\end{array}$} & \multicolumn{2}{|c|}{$\begin{array}{c}\text { Baja } \\
\text { frecuencia }\end{array}$} & \multicolumn{2}{|c|}{$\begin{array}{c}\text { Alta } \\
\text { frecuencia }\end{array}$} & \multicolumn{2}{|c|}{$\begin{array}{c}\text { Baja } \\
\text { frecuencia }\end{array}$} \\
\hline & $\mathrm{M}$ & DS & $\mathrm{M}$ & DS & $\mathrm{M}$ & DS & $\mathrm{M}$ & DS & $\mathrm{M}$ & DS & $\mathrm{M}$ & DS & $\mathrm{M}$ & DS & $\mathrm{M}$ & DS \\
\hline Frecuencia & 101.27 & 81.84 & 2.18 & 1.77 & 99.6 & 45.55 & 1.62 & 2.56 & - & - & - & - & - & - & - & - \\
\hline Longitud & 5.7 & 1.03 & 5.78 & 0.96 & 5.57 & 1 & 6.04 & 0.9 & 5.57 & 1 & 5.96 & 0.85 & 5.57 & 1 & 6 & 0,88 \\
\hline BF_TK & 932.24 & 341.87 & 585.53 & 253.27 & 756.6 & 346.68 & 565.06 & 263.2 & 749.15 & 322.08 & 614.39 & 281.61 & 703.52 & 325.21 & 548.08 & 220.23 \\
\hline BF_TP & 55.14 & 37.86 & 48.92 & 24.06 & 42.87 & 25.28 & 64.2 & 33.32 & 45.52 & 28.83 & 62.61 & 33.31 & 41.81 & 23.84 & 59.18 & 31.12 \\
\hline $\mathrm{N}$ & 3.11 & 2.94 & 3.07 & 2.72 & 2.78 & 2.88 & 2.11 & 1.93 & 3.46 & 3.94 & 2.7 & 1.68 & 3.43 & 3.73 & 2.22 & 1.76 \\
\hline
\end{tabular}


Artículo. Micaela Difalcis, Samanta Leiva, Aldo Ferreres, Valeria Abusamra. Reconocimiento de palabras en español en una tarea de decisión léxica visual con pseudohomófonos

\section{Procedimiento}

La prueba fue suministrada a los participantes de manera individual en una sala silenciosa en una sesión de 15 minutos de duración aproximada. Los estímulos se presentaron de a uno en la pantalla de una laptop con letra fuente Arial tamaño 30. Los participantes se sentaron a una distancia aproximada de $50 \mathrm{~cm}$, se les explicó que iban a aparecer en la pantalla cadenas de letras y que algunas de ellas corresponderían a palabras pertenecientes al español y otras no. De la manera más rápida y precisa posible, deberían presionar la tecla "s" si la cadena correspondía a una palabra del español y la tecla " $n$ " en caso de que no. Previo al suministro de la prueba experimental, se administró el ensayo de práctica para corroborar la comprensión de la consigna.

Para la presentación de los estímulos de la prueba, se conformaron 4 bloques cuyos estímulos fueron distribuidos de manera aleatoria en cada uno. Los bloques 1, 2 y 3 estuvieron compuestos por 56 estímulos cada uno (14 palabras "de relleno", 7 palabras de alta frecuencia, 7 palabras de baja frecuencia, 7 no-palabras de alta frecuencia, 7 no-palabras de baja frecuencia, 7 PSHs de alta frecuencia y 7 PSHs de baja frecuencia). El bloque 4, por 52 estímulos (13 palabras "de relleno", 7 palabras de alta frecuencia, 6 palabras de baja frecuencia, 7 no-palabras de alta frecuencia, 6 nopalabras de baja frecuencia, 7 PSHs de alta frecuencia y 6 PSHs de baja frecuencia). Los bloques fueron presentados a todos los participantes en el mismo orden pero los estímulos dentro de cada bloque fueron randomizados para cada caso particular.

Se registraron los tiempos de reacción en milisegundos y las respuestas de cada participante a través del software SuperLab (Beringer, 1995).

\section{Análisis de datos}

Previo a la realización de los análisis específicos para el testeo de las hipótesis de este trabajo, se realizaron tres análisis centrados en los participantes, los estímulos y los TRs con el fin de excluir aquellos valores atípicos. Para ello, en primer lugar, se calculó el percentil 1 de la cantidad total de aciertos de cada uno los sujetos con el objetivo de excluir del análisis a aquellos participantes cuyo rendimiento fuese igual o menor al mismo. Como resultado, se eliminó del análisis a un participante por su baja cantidad de aciertos $(112 / 165$, percentil $\leq 1)$.

Por otro lado, se calculó el percentil 1 de la cantidad total de aciertos para cada uno de los estímulos agrupados por tipo (palabras, no-palabras y PSHs). Fueron descartados del análisis aquellos estímulos cuyos aciertos fueran igual o menor al 
percentil 1: las palabras "edicto" y "comba", la no-palabra "palario" y el PSH "ganzo". Además, se eliminó el correspondiente PSH ("palasio") de la no-palabra antes mencionada y la correspondiente no-palabra ("ganfo") del PSH antes mencionado.

Respecto de los TRs, no se consideraron 1164 valores $(8.1 \%$ del total) por corresponder a respuestas erróneas de los participantes. Finalmente, se calcularon los percentiles para los TRs restantes agrupados según el tipo de estímulo y se descartaron aquellos iguales o menores al percentil 1 y aquellos iguales o superiores al percentil 98, procediendo por separado con cada tipo de estímulo (palabras, no-palabras, PSHs). Como resultado, fueron excluidos del análisis 394 TRs (3\%) correspondientes a latencias muy largas o muy cortas respecto del rendimiento general en cada caso (para las palabras TRs < $451 \mathrm{msg}$ y > a $2480 \mathrm{msg}$; no-palabras con TRs < $518 \mathrm{msg}$ y > 3057 msg; PSHs con TRs < 499 msg y > 3413 msg).

En relación con el análisis de datos realizado para el testeo de las hipótesis, se calcularon, en primer lugar, los estadísticos descriptivos para la cantidad de errores, así como también para los TRs de cada condición. Luego, para analizar los efectos de lexicalidad, frecuencia y pseudohomofonía sobre los TRs, se realizaron dos análisis centrados en los sujetos $(\mathrm{F} 1)$ y en los ítems $(\mathrm{F} 2)$. En primer lugar, para el análisis F1 se calcularon las medias de los TRs de cada participante para las palabras, no-palabras y pseudohomófonos, cada uno de alta y baja frecuencia. Con esos datos se realizó un ANOVA de medidas repetidas utilizando a las variables tipo de estímulo y frecuencia como factores intra-sujetos y su correspondiente comparación por pares para analizar las diferencias específicas entre cada una de las variables. En segundo lugar, para el análisis F2, se calcularon las medias de los TRs para cada uno de los ítems y se realizó un ANOVA factorial de $3 \times 2$ (tipo de estímulo $\times$ frecuencia) así como los correspondientes análisis post hoc con el DHS de Tukey.

Finalmente, se realizó un ANOVA factorial de 3 x 2 (tipo de estímulo x frecuencia) utilizando la cantidad de errores de cada estímulo como variable dependiente para analizar las diferencias entre la cantidad de errores cometidos por los participantes entre las tres condiciones (palabras versus no-palabras versus PSHs) y para los estímulos de alta y baja frecuencia. Además, se llevaron a cabo los correspondientes análisis post hoc con el DHS de Tukey para analizar las diferencias entre los tres tipos de estímulo. 
Artículo. Micaela Difalcis, Samanta Leiva, Aldo Ferreres, Valeria Abusamra. Reconocimiento de palabras en español en una tarea de decisión léxica visual con pseudohomófonos

\section{Resultados}

\section{Latencias}

En la Tabla 2 se muestran los estadísticos descriptivos para los TRs de las palabras, no-palabras y PSHs de alta y baja frecuencia.

El resultado del ANOVA general mostró diferencias estadísticamente significativas para los TRs $\left(F 2(5)=65.685, p<.001, \eta_{\mathrm{p}}{ }^{2}=.682\right)$. Tanto en los análisis centrados en los sujetos como en los ítems, se observó un efecto principal de frecuencia $\left(F 1(1)=229.736, p<.001, \eta_{\mathrm{p}}{ }^{2}=.721 ; F 2(1)=82.025, p<.001, \eta_{\mathrm{p}}{ }^{2}=.349\right)$ en el que los estímulos de alta frecuencia presentan TRs más cortos $(M=947.3, D E=166.5)$ que los de baja $(M=1097.3, D E=154.1)$. El tamaño del efecto de la influencia de esta variable fue grande para ambos análisis (F1 y F2). Por otro lado, se observó un efecto principal del tipo de estímulo $\left(F 1(2)=115.631, p<.001, \eta_{\mathrm{p}}{ }^{2}=.565 ; F 2(2)=113.57, p<.001, \eta_{\mathrm{p}}{ }^{2}=\right.$ .598) y el análisis del tamaño del efecto mostró una influencia grande de la misma sobre la varianza, tanto para los análisis $\mathrm{F} 1$ como F2. El análisis post hoc y las comparaciones por pares realizadas revelaron que hubo efecto de lexicalidad reflejados tanto en los análisis F1 (Diferencia de medias ${ }_{\mathrm{P} \text { s } \mathrm{NP}}=-255.11, p<.001$ ) como en F2 (DHS de Tukey $\mathrm{P}$ $\left.{ }_{\text {vs } \mathrm{NP}}=-250.1, p<.001\right)$. Como se muestra en la Tabla 2, las palabras tuvieron TRs menores que las no-palabras. Sin embargo, no se halló efecto de pseudohomofonía ni en los análisis centrados en los sujetos (Diferencia de medias $\mathrm{PSH}_{\mathrm{NP}}=15.35, p=.738$ ) ni en aquellos realizados sobre los ítems (DHS de Tukey ${ }_{\mathrm{NP} \text { vs PSH }}=-21.3, p=.527$ ).

Finalmente, se registró un efecto de interacción entre el tipo de estímulo y la frecuencia para ambos análisis $\left(F 1(2)=23.259, p<.001, \eta_{\mathrm{p}^{2}}=.207 ; F 2(2)=5.647, p=\right.$ $\left..004, \eta_{\mathrm{p}}{ }^{2}=.069\right)$. En ambos casos, el efecto de interacción muestra el mismo patrón: los TRs de las no-palabras de alta frecuencia son superiores a los PSH de alta frecuencia, pero se observa el patrón inverso con aquellos de baja frecuencia (los PSH de baja frecuencia tienen TRs mayores a los de las no-palabras de alta frecuencia). En la figura 1 se muestran los TRs comparados para las palabras, no-palabras y los PSHs, así como el patrón de la interacción de estos con la frecuencia. 
Tabla 2. Estadísticos descriptivos de los TRs en milisegundos de las palabras, no-palabras y pseudohomófonos de alta y baja frecuencia.

\begin{tabular}{|c|c|c|c|}
\hline Tipo de estímulo & Frecuencia & $N$ & Media $(D E)$ \\
\hline \multirow[t]{3}{*}{ Palabras } & Alta & 28 & $755.6(35.3)$ \\
\hline & Baja & 25 & 947.5 (110.1) \\
\hline & Total & 53 & 846.1 (124.9) \\
\hline \multirow[t]{3}{*}{ No-palabras } & Alta & 27 & $1062.0(91.0)$ \\
\hline & Baja & 26 & $1131.8(98.2)$ \\
\hline & Total & 53 & $1096.2(100.1)$ \\
\hline \multirow[t]{3}{*}{ Pseudohomófonos } & Alta & 27 & $1031.6(127.2)$ \\
\hline & Baja & 26 & $1206.7(121.6)$ \\
\hline & Total & 53 & $1117.5(151.7)$ \\
\hline \multirow[t]{3}{*}{ Total } & Alta & 82 & $947.3(166.5)$ \\
\hline & Baja & 77 & $1097.3(154.1)$ \\
\hline & Total & 159 & $1020.0(176.8)$ \\
\hline
\end{tabular}
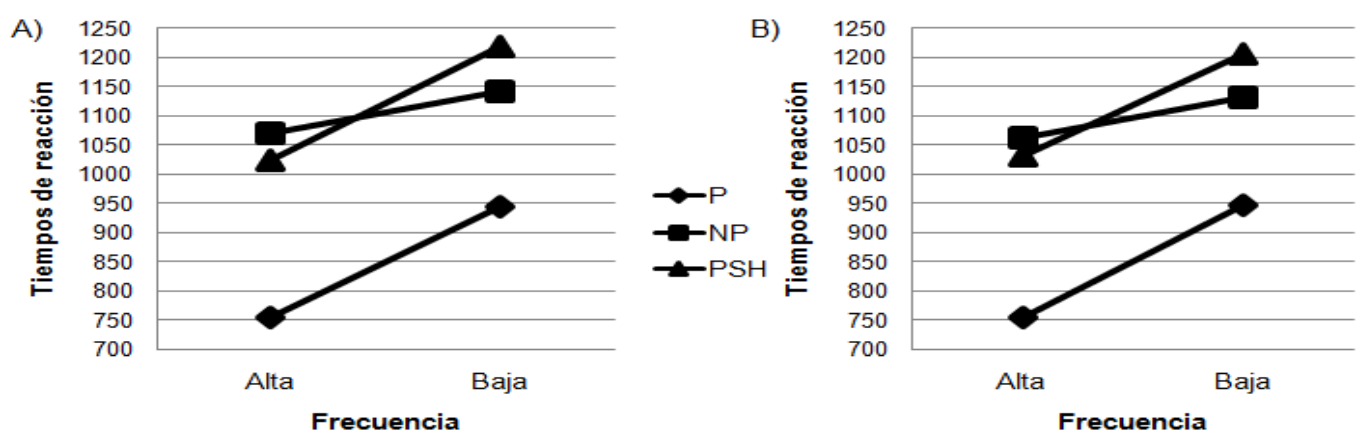

Figura 1. Gráficos de los perfiles de interacción entre el tipo de estímulo (P: palabras; NP: no-palabras; PSH: pseudohomófonos) y la frecuencia sobre los tiempos de reacción (msg) para el análisis F1 (A) y para el análisis F2 (B).

\section{Errores}

En la Tabla 3 se muestran los estadísticos descriptivos de la cantidad de errores de los participantes para las palabras, las no-palabras y los PSHs de alta y baja frecuencia.

El resultado del ANOVA general mostró diferencias estadísticamente significativas para la cantidad de errores cometidos $\left(F(5)=8.929, p<.001, \eta_{\mathrm{p}}{ }^{2}=.226\right)$. Se observó un efecto principal del tipo de estímulo $(F(2)=20.42, p<.001)$ y el análisis del tamaño del efecto mostró una influencia alta de esta variable $\left(\eta_{\mathrm{p}}{ }^{2}=.211\right)$. El análisis post hoc reveló que hubo diferencias estadísticamente significativas entre la cantidad de

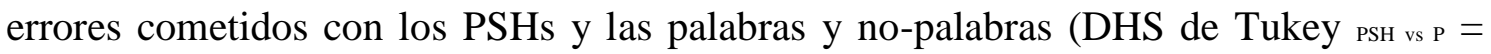
$10.77, p<.001$; DHS de Tukey $\left.\mathrm{PSH}_{\mathrm{v} \mathrm{NP}}=10.47, p<.001\right)$. Los participantes cometieron 
más errores cuando el estímulo presentado era un PSH (total errores $=769$ ) que una palabra (total errores $=198$ ) o no-palabra (total errores $=214$ ). Por otro lado, no hubo diferencias estadísticamente significativas entre la cantidad de errores cometidos con las palabras y las no-palabras (DHS de Tukey ${ }_{\mathrm{p} \text { vs } \mathrm{NP}}=-0.3, p=.987$ ). En la figura 2, se muestran la cantidad de errores totales y los porcentajes para las palabras, no-palabras y PSHs.

No se observó efecto principal de la frecuencia $\left(F(1)=2.857, p=.093, \eta_{\mathrm{p}^{2}}=\right.$ .018) ni de interacción entre las dos variables $\left(F(2)=1.991, p=.140, \eta_{\mathrm{p}}{ }^{2}=.025\right)$ para la cantidad de errores cometidos.

Tabla 3. Estadísticos descriptivos de la cantidad de errores para las palabras, no-palabras y pseudohomófonos de alta y baja frecuencia.

\begin{tabular}{|c|c|c|c|c|c|}
\hline Tipo estímulo & Frecuencia & $n$ & Media de error $(D E)$ & $\begin{array}{c}\text { Cantidad de errores cometidos } \\
\text { por la totalidad de los } \\
\text { participantes }(N=90)\end{array}$ & Porcentaje de error \\
\hline \multirow[t]{3}{*}{ Palabras } & Alta & 22 & $3.2(5.8)$ & 70 & 1.5 \\
\hline & Baja & 31 & $4.1(5.4)$ & 128 & 2.7 \\
\hline & Total & 53 & $3.7(5.6)$ & 198 & 4.2 \\
\hline \multirow[t]{3}{*}{ No-palabras } & Alta & 30 & $4.0(3.5)$ & 121 & 2.5 \\
\hline & Baja & 23 & $4.0(4.0)$ & 93 & 1.9 \\
\hline & Total & 53 & $4.0(3.7)$ & 214 & 4.5 \\
\hline \multirow[t]{3}{*}{ Pseudohomófonos } & Alta & 30 & $11.3(13.4)$ & 339 & 7.1 \\
\hline & Baja & 23 & $18.7(19.8)$ & 430 & 9.0 \\
\hline & Total & 53 & $14.5(16.7)$ & 769 & 16.1 \\
\hline \multirow[t]{3}{*}{ Total } & Alta & 82 & $6.5(9.5)$ & 530 & 11.1 \\
\hline & Baja & 77 & $8.4(13.2)$ & 651 & 13.6 \\
\hline & Total & 159 & $7.4(11.5)$ & 1181 & 24.8 \\
\hline
\end{tabular}

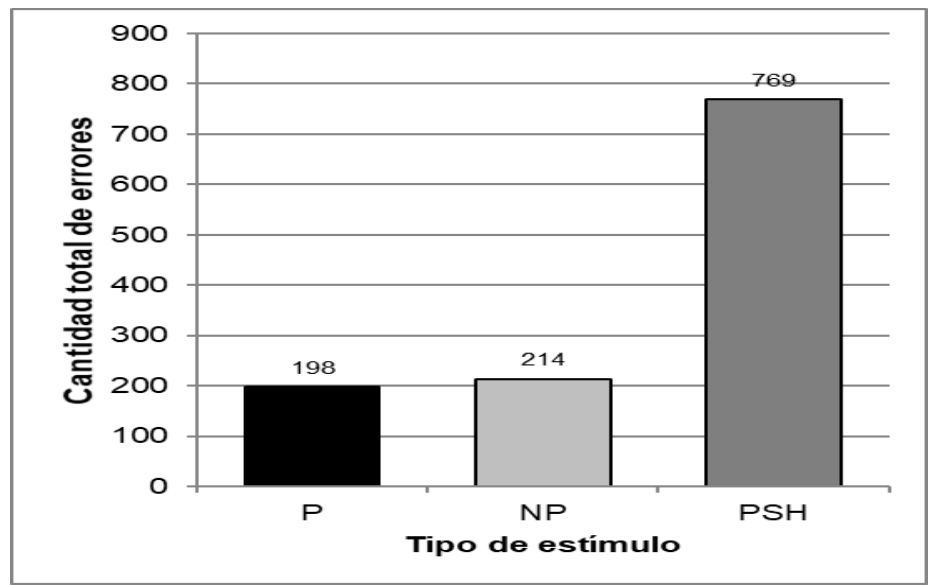

Figura 2. Cantidad de errores totales de los participantes para palabras (P), no-palabras (NP) y pseudohomófonos (PSH). 


\section{Discusión}

El objetivo principal del presente trabajo fue estudiar el efecto de pseudohomofonía en español junto con otras variables psicolingüísticas de interés como la lexicalidad y la frecuencia. Para eso, hemos diseñado una tarea de decisión léxica visual con pseudohomófonos (considerada la más adecuada para el estudio de dicho efecto) en la que se controló y manipuló de manera exhaustiva las variables léxicas y subléxicas de los estímulos seleccionados. Fueron dos los tipos de datos obtenidos que hemos analizado: por un lado, los tiempos de reacción y, por el otro, la tasa de error en el rendimiento de los sujetos.

Respecto del tipo de estímulo, tanto el análisis de los TRs como el de los errores $\mathrm{y}$ aciertos arrojaron diferencias significativas: los participantes presentan menores latencias para palabras que para no-palabras y PSHs (efecto de lexicalidad) y cometen más errores en PSHs que en palabras y no-palabras (efecto de pseudohomofonía). Estos resultados son coincidentes con los hallados en estudios previos llevados a cabo en español (Domínguez y Cuetos, 1992; Monsalve y Cuetos, 2001).

En cuanto al efecto de frecuencia en los TRs, también hallamos diferencias significativas en ambos análisis (F1 y F2) y una interacción con el tipo de estímulo: los PSHs de alta frecuencia presentaron TRs más cortos que los PSHs de baja frecuencia, al igual que lo reportado por Cuetos y Domínguez (2002) en español. Los autores afirman que los PSHs, debido a su similitud fonológica con palabras existentes en la lengua, activan representaciones de dichas palabras en el léxico ortográfico que podrían entrar en competición y lentificar el rechazo del PSH como palabra por parte del sujeto. Estos resultados son coincidentes con el estudio de la frecuencia y la pseudohomofonía en tareas de lectura en voz alta en sujetos angloparlantes (Reynolds y Besner, 2005). El hecho de que los PSHs de alta frecuencia presenten menores latencias que los PSHs de baja frecuencia otorga un rol importante a la fonología en el reconocimiento visual de las palabras. Como ya hemos mencionado, los estímulos del presente trabajo han sido seleccionados y creados bajo un control exhaustivo de variables subléxicas. Si bien esto no se había llevado a cabo con anterioridad en los trabajos realizados en español, nuestros resultados son coincidentes con la literatura.

Por su parte, si bien las latencias de los PSHs son apenas mayores que las de las no-palabras, no se ha observado un efecto significativo de pseudohomofonía en el análisis de los TRs ni en el análisis por sujeto (F1) ni en el análisis por ítem (F2). Es decir, los datos aquí reportados no replican los hallazgos del trabajo precedente en 
lengua española (Cuetos y Domínguez, 2002) debido a que, en él, el efecto de pseudohomofonía encontrado es significativo. Una explicación para esta diferencia podría ser el diseño de los estímulos. Si bien los tipos de estímulo utilizados en la DL con PSHs del presente trabajo y los del mencionado antecedente en español son los mismos (palabras, no-palabras y PSHs y ninguno de los dos experimentos contiene homófonos heterógrafos), la conformación de la lista presenta diferencias entre ambos trabajos debido a las diferencias metodológicas consideradas para la selección y la creación de estímulos: en este trabajo, hemos controlado más variables léxicas (cantidad de vecinos) y subléxicas (frecuencia de bigrama token y type) y hemos modificado una sola letra de las palabras base, en lugar de dos, para construir las no-palabras y los pseudohomófonos. Esto significa que los PSHs de nuestra tarea son más similares a las no-palabras que en el caso de los PSHs utilizados por Cuetos y Domínguez (2002), lo cual podría haber producido que los participantes mostraran TRs más similares entre ambos tipos de estímulo y suprimido el efecto de pseudohomofonía. En futuros trabajos, sería pertinente crear estímulos modificando, al igual que Cuetos y Domínguez (2002), dos letras de las palabras base con el fin de obtener no-palabras y PSHs con formas ortográficas menos similares a las palabras. No solo sería interesante estudiar los TRs, sino también la tasa de error y de acierto de los participantes: los sujetos de nuestro experimento presentaron una tasa de error menor en los PSHs $(16,1 \%)$ que el anterior estudio en lengua española $(18,7 \%)$.

Otra explicación probable de la diferencia de nuestros resultados con lo reportado por trabajos anteriores podría ser que los sujetos utilicen diferentes estrategias de lectura de acuerdo con los tipos de estímulo que conforman la tarea. Varios estudios han demostrado que los diferentes tipos de estímulo que componen las tareas, y cómo se ordenan en bloques, influyen en la estrategia de los participantes al momento de reconocer palabras escritas (McCann y Besner, 1987; Grainger, Spinelli y Ferrand, 2000; Kinoshita, Lupker y Rastle, 2004; Lupker, Brown y Colombo, 1997; Borowsky, Owen y Masson, 2002; Pagliuca et al., 2008; Peresotti y Colombo, 2011). Por ejemplo, los resultados son diferentes cuando las listas están compuestas, además de palabras, no-palabras y PSHs por homófonos heterógrafos. Esto es, en español, existen diferencias entre los pares "bote" y "vote", por un lado, y "bucle" y "vucle", por el otro. Si bien ambos pares comparten la forma fonológica, en el primer caso estamos frente a un par de homófonos heterógrafos (es decir, se trata de dos palabras existentes de la 
lengua) y, en el segundo caso, no debido a que uno de los estímulos ("vucle") es una nopalabra

Por otra parte, el estudio llevado a cabo en el presente trabajo puede contribuir a las investigaciones dedicadas a analizar la funcionalidad de las rutas léxicas y no léxicas de lectura en español en poblaciones clínicas (para una revisión, ver Ferreres y López, 2014).

Respecto de aspectos no abordados en este trabajo, sería muy interesante analizar en futuras investigaciones, debido a que podría explicar las diferencias (sobre todo, en cuanto al efecto de pseudohomofonía) entre los resultados aquí hallados con los datos obtenidos en el estudio realizado previamente en español, la sílaba de cambio para la confección de los PSHs y las no-palabras. Algunas investigaciones llevadas a cabo en otras lenguas han demostrado la importancia de la posición silábica de ciertas propiedades de los estímulos, por ejemplo la "irregularidad" (Rastle y Coltheart, 1999; Álvarez, Carreiras y Perea, 2004; Ripamonti et al., 2018) por lo cual es un factor de relevancia a tener en cuenta. Creemos que la sílaba de cambio podría estar jugando un rol importante en los TRs de la DL visual con PSHs. Estudiar si ésta influye o no en las diferencias entre el rechazo de las no-palabras y los PSHs podría arrojarnos mayor claridad para el análisis del procesamiento cognitivo de la palabra aislada.

Finalmente, y en función de las proyecciones a futuro para el estudio del efecto de pseudohomofonía en español, sería esencial llevar a cabo una tarea de lectura en voz alta de los mismos estímulos de la tarea suministrada en esta investigación para estudiar el efecto de pseudohomofonía y comparar los resultados con los datos obtenidos en decisión léxica. Si bien nuestros resultados son coincidentes con la hipótesis de acceso directo y los resultados del anterior trabajo realizado en lengua española refleja una aparente activación fonológica preléxica (hipótesis de la mediación fonológica), son necesarios más estudios para determinar cabalmente cuál es la participación de la fonología en el reconocimiento visual de palabras en una lengua transparente como el español.

\section{Referencias bibliográficas}

Adelman, James, Sabatos-Devito, M. Marquis, S.y Zachary, E. "Individual differences in reading aloud: A mega-study, item effects, and some models". Cognitive psychology 68 (2014): 113-160. 
Álvarez, Carlos, Carreiras, M. y Perea, Manuel. "Are syllables phonological units in visual word recognition?" Language and Cognitive processes 19.3 (2004): 427452.

Andrews, Sally. "Individual differences in skilled visual word recognition and reading: The role of lexical quality". Visual word recognition. Meaning and context, individuals and development. Hove, England: Psychology Press, 2. 2012.

Barca, Laura, Pezzulo, G., Ouellet, Marc y Ferrand, L. "Dynamic lexical decisions in French: Evidence for a feedback inconsistency effect". Acta psychologica 180 (2017): 23-32.

Berent, Iris y Perfetti, C.A. "A rose is a REEZ: The two-cycle model of phonology assembly in reading English". Psychological Review 102.1 (1995): 146-184.

Beringer, J. Experimental Run Time System [Software]. Frankfurt: BeriSoft, 1995.

Besner, Derek y Davelaar, Eileen. "Suedohomofoan effects in visual word recognition: Evidence for phonological processing”. Canadian Journal of Psychology 37.2 (1983): 300-305.

Borowsky, Ron y Masson, M. "Frequency effects and lexical access: On the interpretation of null pseudohomophone base-word frequency effects". Journal of Experimental Psychology: Human Perception and Performance 25 (1999): 270275.

Borowsky, Ron, William O., y Masson, M. "Diagnostics of phonological lexical processing: Pseudohomophone naming advantages, disadvantages, and base-word frequency effects". Memory \& Cognition 30 (2002): 969-987.

Braun, Mario, Hutzler, F., Münte, T.F., Rotte, M., Dambacher, M., Richlan, F. y Jacobs A. "The neural bases of the pseudohomophone effect: phonological constraints on lexico-semantic access in reading”. Neuroscience 295 (2015): 151-163.

Coltheart, Max, Davelaar, E. Jonasson, J.T. y Besner, D. "Access to the internal lexicon". Attention and Performance. Ed.S. Dornic. London: Academic Press, VI. 1977. 535-555.

Coltheart, M., Perry, C. y Coltheart, Max. "DRC: A computational model of visual word recognition and reading aloud". Psychological Review 108 (2001): 205-256.

Coslett, H. B. "Acquired dislexia". Clinical neuropsychology. New York: Oxford University Press, 2003. 108-125.

Cuetos, Fernando y Domínguez, A. "Efecto de la pseudohomofonía sobre el reconocimiento de palabras en una lengua de ortografía transparente". Psicothema 14.4 (2002): 754-759.

Davis, Colin y Perea, Manuel. "Busca Palabras: A program for deriving orthographic and phonological neighborhood statistics and other psycholinguistic indices in Spanish". Behavior Research Methods 37 (2005): 665-671.

Difalcis, Micaela, Ferreres, Aldo, Osiadacz, Natalia y Abusamra, Valeria. "Latencias de respuesta de lectura en español: efectos de lexicalidad y frecuencia". Investigaciones sobre lectura 9 (2018): 50-72.

Domínguez, Alberto y Cuetos, Fernando. "Desarrollo de las habilidades de reconocimiento de palabras en niños con distinta competencia lectora". Cognitiva 4 (1992): 193-208.

Ellis, Andrew y Young, Andrew. Human cognitive neuropsychology. London: Psychological Press, 1988.

Ferrand, Ludovic y Grainger, Jonathan. "Homophone interference effects in visual word recognition". The Quarterly Journal of Experimental Psychology 56.3 (2003): 403-419. 
Ferreres, Aldo, Cuitiño, Macarena y Olmedo, A. "Acquired surface alexia in Spanish: A case report". Behavioural Neurology 16 (2005): 71-84.

Ferreres, Aldo, López, Cynthia y Fabrizio, Susana. "Alexia de superficie en español sin déficit semántico". Revista Neuropsicología Latinoamericana 4 (2012): 86-103.

Ferreres, Aldo y López. Cynthia "Orthographic transparency and acquired dyslexias (alexias) in Spanish speakers: a review/La transparencia ortográfica y las alexias (acquired dyslexias) en hispanohablantes, una revisión”. Estudios de Psicología 35.3 (2014): 519-544.

Frisson, Steven, Koole, Hannah Hughes, Louisa, Olson, Andrew y Wheeldon, Lind. "Competition between orthographically and phonologically similar words during sentence reading: evidence from eye movements". Journal of Memory and Language 73 (2014): 148-173.

Frost, Ram. "Toward a strong phonological theory of visual word recognition: true issues and false trails". Psychological bulletin 123.1 (1998): 71-99.

Grainger, Jonathan, Spinelli, Elsa y Ferrand, Ludovic. "Effects of baseword frequency and orthographic neighborhood size in pseudohomophone naming". Journal of Memory and Language 42.1 (2000): 88-102.

Hawelka, Stefan, Schuster, Sarah, Gagl Benjamin y Hutzler, Florian "Beyond single syllables: the effect of first syllable frequency and orthographic similarity on eye movements during silent reading". Language and Cognitive Processes 28.8 (2013): 1134-1153.

Kinoshita, Sachiko, Lupker, S. J. y Rastle, K.. "Modulation of regularity and lexicality effects in reading aloud". Memory \& Cognition 32.8 (2004): 1255-1264.

Kuperman, Victor y Van Dyke, Julie. "Effects of individual differences in verbal skills on eye-movement patterns during sentence reading". Journal of Memory and Language, 65 (2011): 42-73.

Kwok Van, Rosa, Cuetos, Fernando, Avdyli, Rrezarta y Ellis, Andrew. "Reading and lexicalization in opaque and transparent orthographies: Word naming and word learning in English and Spanish". Quarterly Journal of Experimental Psychology, 70.10 (2017): 2105-2129.

Lupker, Stephen, Brown, Patrick y Colombo, Lucia. "Strategic control in a naming task: Changing routes or changing deadlines?" Journal of Experimental Psychology: Learning, Memory, and Cognition 23.3 (1997): 570-590.

McCann, Robert y Besner, Derek. "Reading pseudohomophones: Implications for models of pronunciation assembly and the locus of word-frequency effects in naming". Journal of Experimental Psychology: Human Perception and Performance 13.1 (1987): 14-24.

Monsalve, Asunción y Cuetos, F. "Asimetría hemisférica en el reconocimiento de palabras: efectos de frecuencia e imaginabilidad". Psicothema 13.1 (2001): 23-28.

Pagliuca, Giovanni, Arduino, Lisa, Barca, Laura y Burani, Cristina. "Fully transparent orthography, yet lexical reading aloud: The lexicality effect in Italian". Language and Cognitive Processes 23.3 (2008): 422-433.

Perea, Manuel y Rosa, Eva. "Psicología de la lectura y procesamiento léxico visual: Una revisión de técnicas experimentales y procedimientos de análisis". Psicológica, 20 (1999): 65-90.

Peressotti, Francesca y Colombo, Lucía. "Reading aloud pseudohomophones in Italian: Always an advantage." Memory \& Cognition 40.3 (2012): 466-482.

Rastle, Kathleen y Coltheart, Max. "Serial and strategic effects in reading aloud". Journal of Experimental Psychology: Human perception and performance 25.2 (1999): 482-503. 
Reynolds, Michael y Besner, Derek. "Basic processes in reading: A critical review of pseudohomophone effects in reading aloud and a new computational account". Psychonomic Bulletin \& Review 12.4 (2005): 622-646.

Ripamonti, Enrico, Luzzatti, C., Zoccolotti, P y Traficante, D. "Word and pseudoword superiority effects: Evidence from a shallow orthography language". The Quarterly Journal of Experimental Psychology 71.9 (2018): 1911-1920.

Rubenstein, Herbert, Spafford, Lewis y Rubenstein, Mollie. "Homographic entries in the internal lexicon: Effects of systematicity and relative frequency of meanings". Journal of verbal learning and verbal behavior 10.1 (1971): 57-62.

Seidenberg, Mark. "The time course of phonological code activation in two writing systems". Cognition 19.1 (1985): 1-30.

Seidenberg, Mark, Petersen A, MacDonald MC, Plaut, DC. "Pseudohomophone effects and models of word recognition". Journal of Experimental Psychology: Learning, Memory, and Cognition 22.1 (1996): 48-62.

Taft, Marcus y Russell, Bruce. "Pseudohomophone naming and the word frequency effect". The Quarterly Journal of Experimental Psychology Section A 45.1 (1992): $51-71$.

Van Orden, Guy. "A ROWS is a ROSE: Spelling, sound, and reading". Memory \& Cognition 15.3 (1987): 181-198.

Ziegler, Johannes, Arthur, Jacobs y Klüppel, Dorothee . "Pseudohomophone effects in lexical decision: Still a challenge for current word recognition models". Journal of Experimental Psychology: Human Perception and Performance 27.3 (2001): 547-559.

Ziegler, Johannes, Bertrand, D., Lété, B. y Grainger, J. "Orthographic and phonological contributions to reading development: Tracking developmental trajectories using masked priming". Developmental Psychology 50.4 (2014): 1026-1036. 
Ap éndice li: estimulos administrados en h tarea de decisión lérica risual con pseudohomófonos

\begin{tabular}{|c|c|c|c|c|c|c|c|c|c|c|c|c|c|}
\hline & & & & & & ALABRAS & SBASE / & OO-PALAE & BRAS/ PSEUD & OHOMOFON & & & \\
\hline & PALA & IBRAS & & & Alth fre & ecuracia & & & & Baja ff & fecuencia & & \\
\hline Alta frecuencia & Bloque & Baja frecuencia & Blogve & Palabra base & No-palabras & Bloque & PSH & Bloque & Palabra bass & Nopalabras & Bloque & PSH & Bloque \\
\hline actind & 1 & Acelga & 1 & abvalo & apvelo & 4 & awelo & 2 & amaba & amoda & 2 & mava & 2 \\
\hline amigo & 1 & Aditivo & 1 & asurto & atunto & 1 & azunto & 3 & angina & anpina & 1 & snjina & 3 \\
\hline autor & 1 & Almenda & 4 & barrio & tario & 2 & vario & 2 & bache & rache & 3 & vache & 3 \\
\hline calor & 3 & Allas & 4 & base & bane & 4 & bace & 1 & barrote & parrote & 2 & varote & 4 \\
\hline came & 4 & Barbero & 1 & brazo & brago & 3 & braso & 1 & bastra & matusa & 3 & vabta & 3 \\
\hline color & 3 & Crrpa & 1 & cerebro & verebro & 1 & serebro & 1 & boceto & bodeto & 1 & boset b & 1 \\
\hline esposa & 3 & Cotcha & 2 & cine & ting & 1 & sing & 4 & boquete & poquete & 3 & voquate & 2 \\
\hline fiesta & 4 & Comba & 2 & especie & espenis & 3 & espesie & 4 & bozal & boal & 3 & bosal & 2 \\
\hline fvego & 3 & Dadal & 1 & gente & pente & 4 & jente & 3 & bude & mocle & 4 & wele & 4 \\
\hline futhro & 2 & Bdicto & 2 & gesto & festo & 3 & jesto & 1 & cabina & catina & 3 & avina & 1 \\
\hline hotel & 3 & Flate & 1 & juicio & juitio & 3 & juisio & 2 & $\operatorname{cep} 0$ & mapo & 3 & $s p 0$ & 1 \\
\hline inviemo & 2 & Gancho & 2 & mariz & rarij & 4 & maris & 3 & cocel & coffel & 3 & consel & 3 \\
\hline leche & 2 & Lagasto & 3 & nivel & nisal & 3 & nibel & 3 & forraje & fomape & 4 & forrage & 3 \\
\hline lectura & 2 & Liebre & 3 & noticia & notifia & 1 & notisis & 4 & frazada & frabada & 1 & frasada & 4 \\
\hline materia & 4 & Maceta & 4 & sovelas & nomala & 2 & noboela & 3 & galleta & gafeta & 4 & gayeta & 1 \\
\hline marcado & 1 & Novato & 2 & palacio & palario & 1 & palasio & 1 & ganso & ganfo & 2 & ganzo & 2 \\
\hline mirads & 2 & Ojera & 3 & pasitlo & parillo & 1 & pacillo & 4 & gana & gama & 3 & gara & 3 \\
\hline momanto & 2 & Panal & 3 & plaza & plaja & 3 & plass & 2 & glaciar & glafir & 1 & glasiar & 1 \\
\hline perro & 4 & Pedal & 2 & precio & pretio & 3 & presio & 2 & laringe & larings & 1 & Ixinje & 1 \\
\hline piedra & 4 & Pum & 1 & prens & prenca & 2 & prenza & 2 & lechuza & lechuja & 4 & lechva & 1 \\
\hline poeta & 1 & Retazo & 4 & sala & nala & 1 & zala & 4 & Havero & llatero & 1 & Ilabero & 4 \\
\hline radio & 4 & Serador & 4 & sald & ralud & 2 & zalud & 4 & mora & morpa & 2 & mona & 3 \\
\hline ropa & 1 & Talco & 3 & gerie & ferie & 2 & cerie & 4 & praje & parave & 2 & parage & 2 \\
\hline sombra & 4 & lenador & 3 & sigglo & diglo & 2 & ciglo & 3 & poitlla & polida & 2 & poliyg & 2 \\
\hline suelo & 3 & tomo & 4 & valor & ralor & 4 & balor & 1 & tocayo & tocafo & 4 & tocallo & 2 \\
\hline temento & 3 & vaquezo & 2 & ventana & pentana & 2 & bentura & 3 & velero & jelaro & 4 & beleso & 4 \\
\hline texto & 2 & zamba & 3 & viento & riento & 4 & biento & 2 & veletas & peleta & 1 & beleta & 4 \\
\hline tono & 1 & & & $20 \mathrm{an}$ & pona & 4 & $800 a$ & 1 & & & & & \\
\hline
\end{tabular}

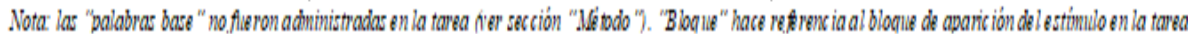

